(C) The Author(s), 2020. Published by Cambridge University Press on behalf of The Nutrition Society. This is an Open Access article, distributed under the terms of the Creative Commons Attribution licence (http://creativecommons.org/licenses/by/4.0/), which permits unrestricted re-use, distribution, and reproduction in any medium, provided the original work is properly cited.

\title{
Effect of oral or intragastric delivery of the bitter tastant quinine on food intake and appetite sensations: a randomised crossover trial
}

\author{
Tim Klaassen ${ }^{1,2 *}$, Daniel Keszthelyi ${ }^{2}$, Annick M. E. Alleleyn ${ }^{2}$, Ellen Wilms ${ }^{2}$, Aalt Bast ${ }^{1}$, Adrian A. M. Masclee $^{2}$ \\ and Freddy J. Troost ${ }^{1,2}$ \\ ${ }^{1}$ Food Innovation and Health, Centre for Healthy Eating and Food Innovation, Maastricht University, 5911 AA Venlo, The \\ Netherlands \\ ${ }^{2}$ Division of Gastroenterology-Hepatology, Department of Internal Medicine, School of Nutrition and Translational Research \\ in Metabolism (NUTRIM), Maastricht University Medical Centre, 6202 AZ Maastricht, The Netherlands
}

(Submitted 8 April 2020 - Final revision received 19 June 2020 - Accepted 4 July 2020 - First published online 14 July 2020)

\section{Abstract}

Stimulation of gastrointestinal taste receptors affects eating behaviour. Intraduodenal infusion of tastants leads to increased satiation and reduced food intake, whereas intraileal infusion of tastants does not affect eating behaviour. Currently, it is unknown whether oral- or intragastric administration of tastants induces a larger effect on eating behaviour. This study investigated the effects of oral-and/or intragastric administration of quinine on food intake, appetite sensations and heart rate variability (HRV). In a blinded randomised crossover trial, thirty-two healthy volunteers participated in four interventions with a 1-week washout: oral placebo and intragastric placebo (OPGP), oral quinine and intragastric placebo (OQGP), oral placebo and intragastric quinine (OPGQ) and oral quinine and intragastric quinine (OQGQ). On test days, 150 min after a standardised breakfast, subjects ingested a capsule containing quinine or placebo and were sham-fed a mixture of quinine or placebo orally. At 50 min after intervention, subjects received an ad libitum meal to measure food intake. Visual analogue scales for appetite sensations were collected, and HRV measurements were performed at regular intervals. Oral and/or intragastric delivery of the bitter tastant quinine did not affect food intake (OPGP: 3273.6 (SEm 131.8) kJ, OQGP: $3072 \cdot 7$ (SEM 132.2) kJ, OPGQ: $3289 \cdot 0$ (sem 132.6) kJ and OQGQ: 3204.1 (sEM 133.1) kJ, $P=0.069)$. Desire to eat and hunger decreased after OQGP and OPGQ compared with OPGP $(P<0.001$ and $P<0.05$, respectively), whereas satiation, fullness and HRV did not differ between interventions. In conclusion, sole oral sham feeding with and sole intragastric delivery of quinine decreased desire to eat and hunger, without affecting food intake, satiation, fullness or HRV.

Key words: Bitter tastants: Food intake: Appetite sensations: Oral sham feeding: Intragastric administration

Since 1975, the prevalence of obesity has nearly tripled, being a major healthcare problem worldwide ${ }^{(1)}$, urging the need for non-invasive treatment strategies. The gastrointestinal (GI) tract plays a crucial role in the regulation of eating behaviour via its ability to produce and secrete GI peptides upon foodderived triggers ${ }^{(2-4)}$.

In order for the GI tract to modulate eating behaviour, food constituents need to be sensed in the gut. It has been shown that intestinal infusion of macronutrients reduces food intake that is accompanied by the release of GI peptides, generally called the intestinal brake ${ }^{(5-7)}$, which is operable in a proximal to distal intestinal gradient ${ }^{(8)}$.

Another way to perceive food constituents in the gut is via taste receptors, which are expressed throughout the entire GI $\operatorname{tract}^{(9-11)}$ and have been shown to trigger the release of GI peptides in vitro ${ }^{(12-15)}$. Duodenal delivery of a tastant mixture containing non-energetic sweet, bitter and umami tastants resulted in significant decrease in food intake, without affecting systemic GI peptide levels ${ }^{(16)}$. However, the proximal to distal gradient operable for macronutrients was not applicable to $\operatorname{tastants}^{(17)}$

Inhibition of food intake via taste receptors may occur via pathways different from those via nutrient receptors. It has been hypothesised that taste predicts the type of food that is ingested (i.e. bitter for toxic substances, umami for amino acids and sweet for carbohydrates) ${ }^{(18)}$. Since bitter taste is linked to toxic substances, subsequent food intake could be limited via an aversive reaction to bitter tastants. Currently, data on sole oral effects of (bitter) tastant delivery (i.e. without ingesting) on eating behaviour are lacking.

Abbreviations: ANS, autonomic nervous system; GI, gastrointestinal; HRV, heart rate variability; OPGP, oral placebo and intragastric placebo; OPGQ, oral placebo and intragastric quinine; OQGP, oral quinine and intragastric placebo; OQGQ, oral quinine and intragastric quinine; VAS, visual analogue scale.

* Corresponding author: Tim Klaassen, email t.klaassen@maastrichtuniversity.nl 
Besides endocrine signalling, the autonomic nervous system (ANS) plays a role in appetite signalling. Changes in the balance of ANS can occur prior to changes in GI peptide levels in the presence of a shift from hunger to satiety ${ }^{(19,20)}$. Increased sympathetic tone is suggested to indicate satiation in humans ${ }^{(21)}$. In addition to aversive responses in reaction to bitter substances, bitter taste also exerts a strong sympathetic activation ${ }^{(22)}$. A simple, non-invasive method to measure ANS is heart rate variability (HRV), a physiological variation in time interval between consecutive heartbeats ${ }^{(23)}$.

This study investigated the effects of oral- $v$. intragastric $v$. synchronous oral- and intragastric delivery of the bitter tastant quinine on eating behaviour in healthy subjects, with ad libitum food intake as primary outcome and HRV, appetite sensations and GI-complaints as secondary outcomes. We hypothesised that synchronous oral and intragastric delivery of quinine decreases food intake compared with placebo or delivery of quinine solely oral- or intragastric. Moreover, we expected that solely oral- or intragastric delivery of quinine would decrease food intake compared with placebo, albeit to a lesser extent than synchronous oral- and intragastric delivery of quinine. Finally, we expect oral delivery of quinine to decrease food intake to a greater extent than intragastric delivery of quinine.

\section{Methods}

This study was approved by the Medical Ethics Committee of the Maastricht University Medical Center+ (MUMC+), Maastricht, the Netherlands (ID METC163047), and performed in full accordance with the Declaration of Helsinki (latest amendment by the World Medic Association in 2013) and Dutch Regulations on Medical Research involving Human Subjects (WMO, 1998). The study was performed at the Maastricht University Campus Venlo from 17 August 2018 until 15 August 2019. This study was registered in the US National Library of Medicine (http:// www.clinicaltrials.gov, ID NCT 03565133). All subjects gave written informed consent before screening.

\section{Subjects}

Healthy men and women were recruited by local advertisements. Inclusion criteria were age between 18 and 65 years, a BMI between 18 and $25 \mathrm{~kg} / \mathrm{m}^{2}$, with a stable weight over the past 6 months ( $<5 \%$ body weight change). Exclusion criteria were GI complaints, history of chronic or severe disease, use of medication influencing study endpoints within $14 \mathrm{~d}$ prior to testing, administration of investigational drugs which interfere with this study, major abdominal surgery, dieting, pregnancy or lactation, excessive alcohol consumption ( $>20$ alcoholic consumptions per week), smoking and non-tasters of the bitter stimulus quinine.

Prior to testing, screening was performed where abovementioned in- and exclusion criteria were checked and a taste perception test with quinine was performed. Subjects tasted quinine $(0.5 \mathrm{mmol} / \mathrm{l})$ and tap water blindly and had to indicate their sense of taste. Subjects had to correctly identify the bitter taste of the quinine sample in order to be eligible for the study. Furthermore, length and weight were measured to calculate BMI.

\section{Study design}

In this blinded randomised, placebo-controlled crossover study, subjects received a capsule containing quinine or placebo (cellulose, which is tasteless and odourless in itself ${ }^{(22,24)}$ ) and subjects performed oral sham-feeding with quinine dissolved in tap water or placebo (tap water) on four test days separated by at least 1-week wash-out. An independent researcher not involved in execution of the study generated the randomisation list using www.sealedenvelope.com. On each test day, participants received one of the following interventions in random order: oral placebo and intragastric placebo (OPGP), oral quinine and intragastric placebo (OQGP), oral placebo and intragastric quinine (OPGQ), and oral quinine and intragastric quinine (OQGQ). Subjects and researchers were blinded for the containments of the capsule, but not for the substance used for oral sham feeding as subjects cannot be blinded for the taste of oral sham feeding intervention. Moreover, due to an evolutionary aversive reaction to tasting a bitter substance ${ }^{(25)}$, subjects would involuntarily show whether oral sham feeding with tap water or with quinine dissolved in tap water is being performed. Therefore, the researcher could not be blinded for the oral sham condition.

\section{Oral sham feeding}

For oral sham feeding, $75 \mathrm{mg}$ of quinine-hydrochloride (Arnold Suhr) was dissolved in $120 \mathrm{ml}$ tap water. A magnetic stirrer was used to dissolve the quinine. As the placebo condition, $120 \mathrm{ml}$ tap water were used. Sham feeding was performed by letting subjects take several sips (habitual size as if they would take a sip of a drink) out of one cup and letting them swish the contents around with closed mouth for 20-30 s before expectorating it in a different cup according to previously described protocols ${ }^{(26,27)}$. This induced 2-3 min of sensory stimulation.

\section{Intragastric capsule}

Capsules were manufactured by 'Verenigde Apotheken Limburg (VAL) Bereidingsapotheek'. Gelatin capsules were filled with $75 \mathrm{mg}$ quinine-hydrochloride and cellulose. As the placebo, capsules were filled with cellulose only. The capsules were indistinguishable in terms of appearance and weight. According to the VAL capsule, breakup time was $<9$ min (unpublished results). A dosage of $75 \mathrm{mg}$ quinine-hydrochloride was used, as this aligns with previous research from our group ${ }^{(16,17)}$.

\section{Protocol}

On each test day, after an $8 \mathrm{~h}$ overnight fast, subjects arrived at 08.30 hours at the Maastricht University Campus Venlo. Subjects were instructed to consume the same habitual meal on the evening prior to testing. At $t=-150 \mathrm{~min}$, subjects consumed a standardised liquid breakfast meal ( $250 \mathrm{ml}$ Goedemorgen drinkontbijt (Vifit, Friesland Campina); energy $649 \mathrm{~kJ}$ ( $155 \mathrm{kcal})$ per portion, $22 \mathrm{~g}$ carbohydrates, $8.5 \mathrm{~g}$ protein, and $2 \mathrm{~g}$ fat). At $150 \mathrm{~min}$ (at $t=0 \mathrm{~min}$ ) after breakfast consumption, subjects ingested the capsule with $120 \mathrm{ml}$ tap water and directly thereafter performed the oral sham feeding. Participants were not allowed to rinse their mouths after the sham feeding. Participants 


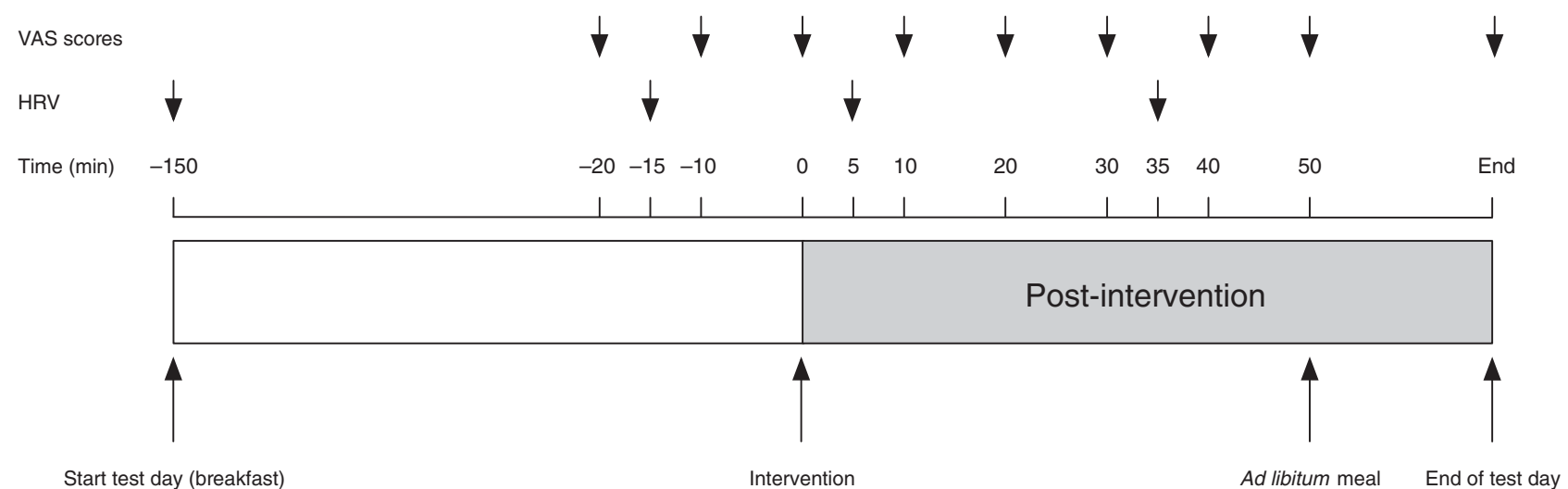

Fig. 1. Timeline of the test day. A standardised breakfast was consumed at $t=-150 \mathrm{~min}$. At $t=0 \mathrm{~min}$, participants ingested a capsule containing quinine or placebo and oral sham feeding with quinine dissolved in tap water or solely tap water was performed. At 50 min after the intervention, an ad libitum test meal was offered. Heart rate variability (HRV) measurements were performed, and visual analogue scales (VAS) scores for appetite sensations and gastrointestinal complaints were collected over the course of the test day. Endpoint of the study and last VAS scores varied per subject, depending on the duration of test meal intake.

reported a persistent bitter taste in their mouth, although this was not quantified. At $50 \mathrm{~min}$ after the intervention (at $t=50$ ), subjects received a standardised ad libitum lunch meal. During the test day, HRV measurements (at $t=-150 \mathrm{~min}, t=-15 \mathrm{~min}$, $t=5 \mathrm{~min}$, and $t=35 \mathrm{~min}$ ) were performed and visual analogue scale (VAS) scores for appetite sensations $(t=-20 \mathrm{~min}$, $t=-10 \mathrm{~min}, \quad t=0 \mathrm{~min}, \quad t=10 \mathrm{~min}, \quad t=20 \mathrm{~min}, \quad t=30 \mathrm{~min}$, $t=40 \mathrm{~min}, t=50 \mathrm{~min}$, and $t=\mathrm{END})$ were collected. An overview of the timeline of the test days can be found in Fig. 1.

\section{Ad libitum test meal}

Subjects received a standardised ad libitum lunch meal (Lasagna Bolognese (Plus supermarket); energy density per $100 \mathrm{~g}: 582 \mathrm{~kJ}$ $(139 \mathrm{kcal})$; food constituents: $10.6 \mathrm{~g}$ carbohydrates, $8.1 \mathrm{~g}$ protein, and $6.7 \mathrm{~g}$ fat) $50 \mathrm{~min}$ (at $t=50 \mathrm{~min}$ ) after the intervention. The test meal was offered in excess (a portion of $1 \mathrm{~kg}(5816 \mathrm{~kJ}$ $(1390 \mathrm{kcal})$ ), which is more than half of the recommended daily intake. No participant finished the entire portion), and subjects were instructed to eat until they felt satiated.

\section{Visual analogue scales for appetite sensations and gastrointestinal complaints}

Appetite sensations and GI complaints (e.g. satiety, hunger, stomach pain and nausea) were measured using VAS (0-100 mm) over the course of the test day. Subjects were asked to indicate on a line, anchored at the low end with the lowest intensity feelings, with opposing terms at the high end, which place on the scale best reflected their feeling at that moment ${ }^{(28)}$.

\section{Heart rate variability}

Utilising only standard and unipolar leads, short time electrocardiogram records were obtained using computer electrocardiograph 'Poly-Spectrum-8/E' (Neurosoft $\circledast$, version 5.3.1.0). Recordings of $5 \mathrm{~min}$ were performed after a resting period of at least $5 \mathrm{~min}$. Measurements were performed in a semirecumbent position in order to minimise ectopic activity. With a sampling frequency of $2 \mathrm{kHz}$, successive heartbeats or inter-beat intervals were determined and analysed according to HRV standards using 'Poly-Spectrum- 8 ' (Neurosoft $\circledast$, version 5.3.1.0) software ${ }^{(23)}$. The power spectral analysis was performed with a fast Fourier transformation. Total power $(0 \cdot 01-0.40 \mathrm{~Hz})$, high-frequency power $(0 \cdot 15-0 \cdot 40 \mathrm{~Hz})$, lowfrequency power $(0 \cdot 04-0 \cdot 15 \mathrm{~Hz})$, and very low-frequency power $(0 \cdot 01-0.04 \mathrm{~Hz})$ were calculated. For this study, heart rate and low-frequency:high-frequency ratio were investigated.

\section{Statistical analyses}

A sample size calculation was based on the difference in food intake after duodenal infusion of the bitter tastant quinine in comparison to food intake after duodenal infusion of placebo as reported by van Avesaat et al. ${ }^{(16)}$. We anticipated the same magnitude for change comparing the combined oral and gastric administration $v$. placebo. Using a difference in means of $184 \mathrm{~kJ}$ ( $44 \mathrm{kcal}$ ), a standard deviation of difference of $356 \mathrm{~kJ}$ ( $85 \mathrm{kcal}$ ), a power of $80 \%$, and an $\alpha$ of $5 \%$, a total number of thirty-two subjects was needed.

Data were analysed using IBM SPSS Statistics 25 (IBM Corporation). Kolmogorov-Smirnov tests and a visual check of normality of the data were performed. The primary outcome of this study was food intake during an ad libitum lunch meal. Secondary outcomes were VAS scores for appetite sensations and GI complaints, and HRV measurements. Data are presented as mean values with their standard errors (unless specified otherwise).

Age, BMI and sex were calculated by descriptive statistics.

Food intake measurements were compared using an autoregressive (1) linear mixed model with intervention, test day and the interaction of intervention $\times$ test day as fixed factors. In case, no intervention $\times$ test day interaction was found, this factor was removed from the model to get the best model fit.

VAS scores for appetite sensations and GI complaints were compared using an autoregressive (1) linear mixed model with intervention, test day, time and the interactions of intervention $x$ test day and time $x$ intervention as fixed factors. In case no intervention $\times$ test day interaction was found, this factor was removed from the model to get the best model fit. 
HRV measurements were compared using an autoregressive (1) linear mixed model with intervention, test day, time and the interactions of intervention $\times$ test day and time $\times$ intervention as fixed factors. In case no intervention $\times$ test day interaction was found, this factor was removed from the model to get the best model fit.

For all analyses, $P<0.05$ was considered statistically significant. Whenever a main effect was found, Bonferroni correction for multiple testing was performed per outcome parameter.

\section{Results}

Subjects

In total, thirty-four subjects met the in- and exclusion criteria. Two subjects dropped out after the first test day due to personal circumstances. Therefore, thirty-two healthy volunteers (twentyfour female, age 25.3 (SD 3.8) years, BMI 22.5 (SD 1.9) kg/m²) completed the study protocol and were included in the analyses. No other adverse events were reported, and all subjects tolerated the interventions well.

\section{Food intake}

Based on estimated means, no differences in ad libitum food intake were observed after oral sham feeding with quinine, intragastric delivery of quinine or combined oral sham feeding withand intragastric delivery of quinine $v$. placebo (placebo: 3273.6 (SEM 131.8) kJ, oral sham feeding with quinine: $3072 \cdot 7$ (SEM 132.2) $\mathrm{kJ}$, intragastric delivery of quinine: 3289.0 (SEM 132.6) kJ, oral sham feeding with- and intragastric delivery of quinine: $3204 \cdot 1$ (sem 133.1) kJ, $P=0.069$ ) (Fig. 2). Furthermore, no trends in individual responses were found (Fig. 3).

\section{Appetite sensations}

The mean VAS scores for desire to eat, hunger, satiation and fullness are depicted in Fig. 4. Based on a linear mixed model, significant main effects of intervention were found for desire to eat and hunger scores, indicating an overall intervention effect on these scores. Post hoc pairwise comparisons showed a significant reduction in desire to eat and hunger scores for oral sham feeding with quinine $v$. placebo (both $P<0.001$ ), intragastric delivery of quinine $v$. placebo (both $P<0.05$ ), and oral sham feeding with quinine $v$. oral sham feeding with- and intragastric delivery of quinine (both $P<0 \cdot 01$ ). No main effects of intervention were found for satiation and fullness scores. No intervention $x$ time interactions were found for any of the VAS scores; thus, intervention effects did not differ on specific time points.

\section{Gastrointestinal complaints}

The mean VAS scores for stomach pain, bloating and nausea are depicted in Fig. 5. No intervention effects for these VAS scores were found. Moreover, no intervention $\times$ time point interactions were found for these VAS scores.
Food intake

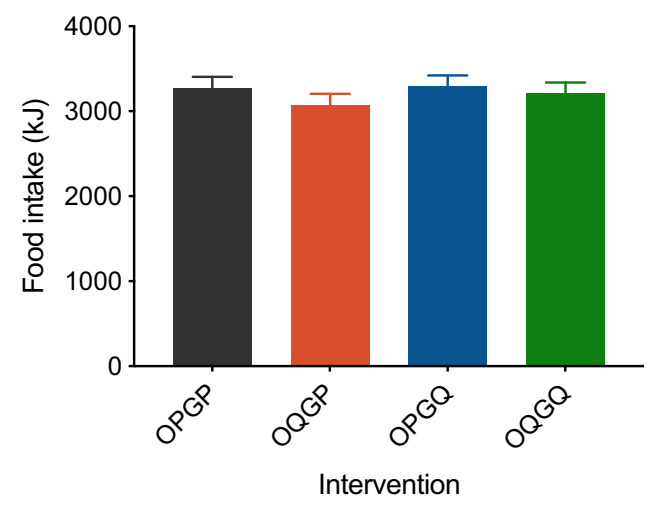

Fig. 2. Amount eaten in $\mathrm{kJ}$ (mean + SEM) 50 min after oral placebo and intragastric placebo (OPGP), oral quinine and intragastric placebo (OQGP), oral placebo and intragastric quinine (OPGQ) and quinine both oral and intragastric (OQGQ). Based on an autoregressive (1) linear mixed model, no difference in food intake was observed between the conditions $(P=0.069)$. OPGP, , OQGP, ,OPGQ, , OQGQ.

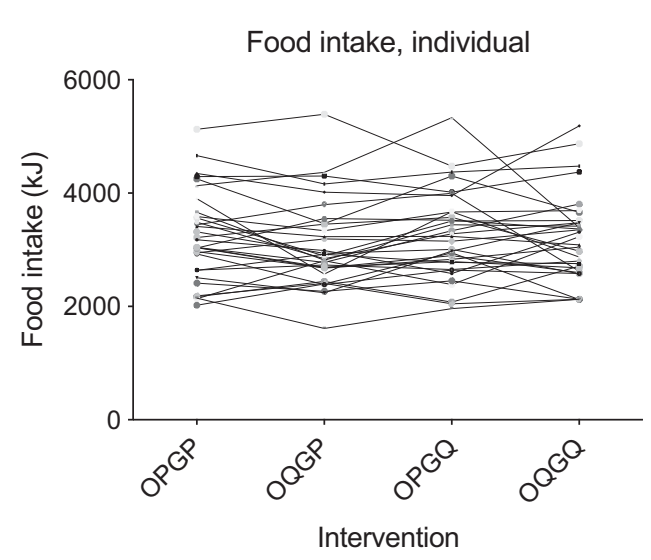

Fig. 3. Individual representation per subject of amount eaten in $\mathrm{kJ}$ (mean + SEM) $50 \mathrm{~min}$ after oral placebo and intragastric placebo (OPGP), oral quinine and intragastric placebo (OQGP), oral placebo and intragastric quinine (OPGQ) and quinine both oral and intragastric (OQGQ). Based on an autoregressive (1) linear mixed model, no difference in food intake was observed between the conditions $(P=0.069)$.

\section{Heart rate variability}

Heart rate and low-frequency:high-frequency ratio are depicted in Fig. 6. No intervention effects for these HRV measurements were found. Moreover, no intervention $\times$ time point interactions were found for these HRV measurements.

\section{Discussion}

Our results show that food intake is affected neither by oral sham feeding of quinine, nor intragastric delivery of quinine, nor synchronous oral sham feeding and intragastric delivery of quinine compared with placebo. Moreover, no differences have been found in VAS scores for satiation or fullness and HRV measurements. However, VAS scores for desire to eat and hunger decreased significantly after oral sham feeding and intragastric delivery of quinine. 
(a)

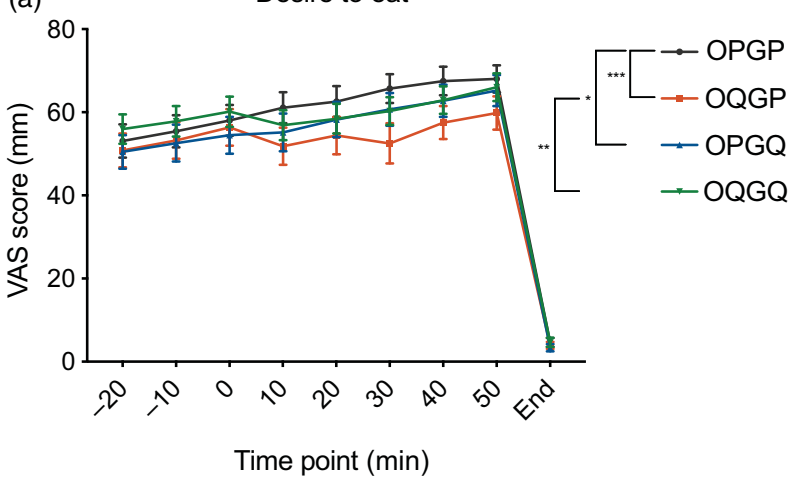

(b) Hunger

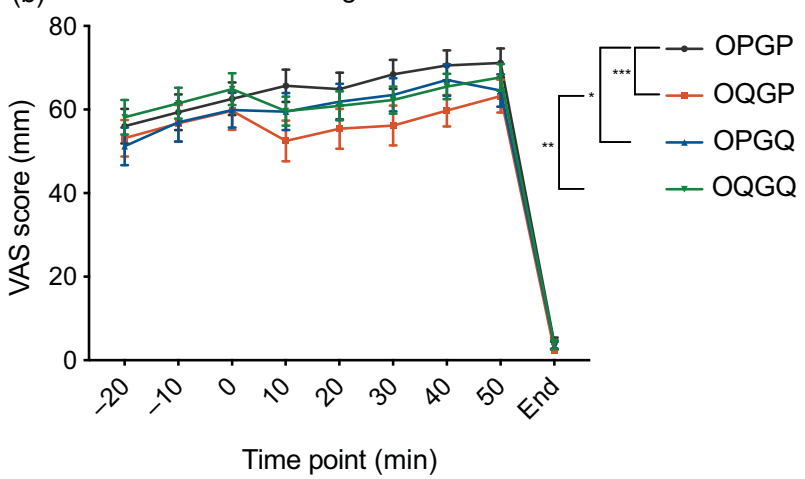

(d)

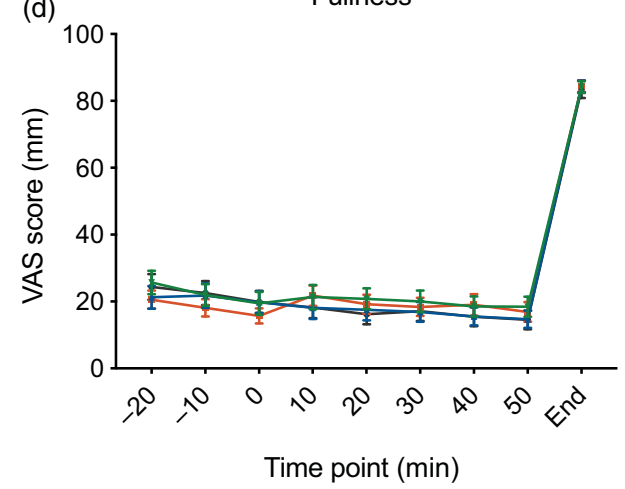

Fig. 4. Visual analogue scale (VAS) scores for desire to eat (a), hunger (b), satiation (c) and fullness (d) (mean + SEM) before and after oral placebo and intragastric placebo (OPGP), oral quinine and intragastric placebo (OQGP), oral placebo and intragastric quinine (OPGQ) and quinine both oral and intragastric (OQGQ). Based on an autoregressive (1) linear mixed model, intervention effects were found for desire to eat and hunger scores $(P<0.001)$. Bonferroni correction was applied for post hoc pairwise comparisons. ${ }^{*} P<0.05,{ }^{\star *} P<0.01,{ }^{* * *} P<0.001 . \rightarrow$, OPGP $\rightarrow$, OQGP,$\longrightarrow$, OPGQ, $\rightarrow$, OQGQ.

To the best of our knowledge, the effects of oral sham feeding with (bitter) tastants have not been described in literature previously. Moreover, the effects of bitter tastants on eating behaviour have been conducted only in small-sized studies. This is the first study to investigate the acute effects of a bitter tastant on eating behaviour in an adequately sized study.

The results of studies on the effects of bitter tastants on food intake, appetite sensations and GI peptides are not conclusive. Most studies reported only a trend towards a decrease in food intake after intragastric or intraduodenal delivery of bitter tastants ${ }^{(16,29-32)}$. Some studies, however, reported a statistically significant reduction in food intake ${ }^{(33,34)}$. In one study, no initial effect of encapsulated bitter compounds on food intake was observed, while over the day a significant decrease in food intake was found ${ }^{(35)}$. Deloose et al. ${ }^{(29)}$ showed a longer sustained satiety effect after a standardised meal when the meal was preceded by intragastric administration of denatonium benzoate. Taken together, these results indicate that delivery of bitter tastants in the presence of, or followed by a energetic content, exerts a more pronounced effect on eating behaviour. With respect to our study, it is possible that a more marked decrease in food intake could have been found if food intake was measured over the course of the day. However, our aim was to explore in detail the acute effects of oral sham feeding and/or intragastric delivery of the bitter tastants quinine on energetic intake during the next meal and not over a longer period.
Intragastric administration of bitter compounds (quinine and denatonium benzoate) has been shown to reduce antral motility ${ }^{(29,36)}$ and to decrease systemic motilin and ghrelin levels ${ }^{(29,34,36)}$, whereas effects of bitter compounds on overall gastric emptying rate have not been demonstrated ${ }^{(29,32,33,37)}$. However, the effects of GI delivery of tastants on circulating GI peptide levels have not been fully elucidated. Several studies did not observe any changes in glucagon-like peptide-1, polypeptide YY and/or cholecystokinin ${ }^{(16,30,32)}$. In one study, an increase in plasma cholecystokinin was observed after intraduodenal delivery of the bitter tastant quinine, but systemic ghrelin levels were not affected ${ }^{(33)}$.

Where systemic GI peptide levels are considered to be an objective parameter to predict prospective food intake, VAS scores for appetite sensations can give insight in subjective feelings relating to prospective food intake. In the present study, oral sham feeding or intragastric delivery of quinine decreased the desire to eat and hunger sensations compared with placebo. Interestingly, there seems to be an overall trend of an upward slope for appetite throughout the test day. In this study, subjects were provided with the test meal around noon. Therefore, this might reflect the natural tendency to get an increased appetite towards habitual time of meal intake, due to conditioned hunger $^{(38)}$. Important to note is that synchronous oral sham feeding with- and intragastric delivery of quinine did not alter desire to eat and hunger scores compared with placebo. This rather 

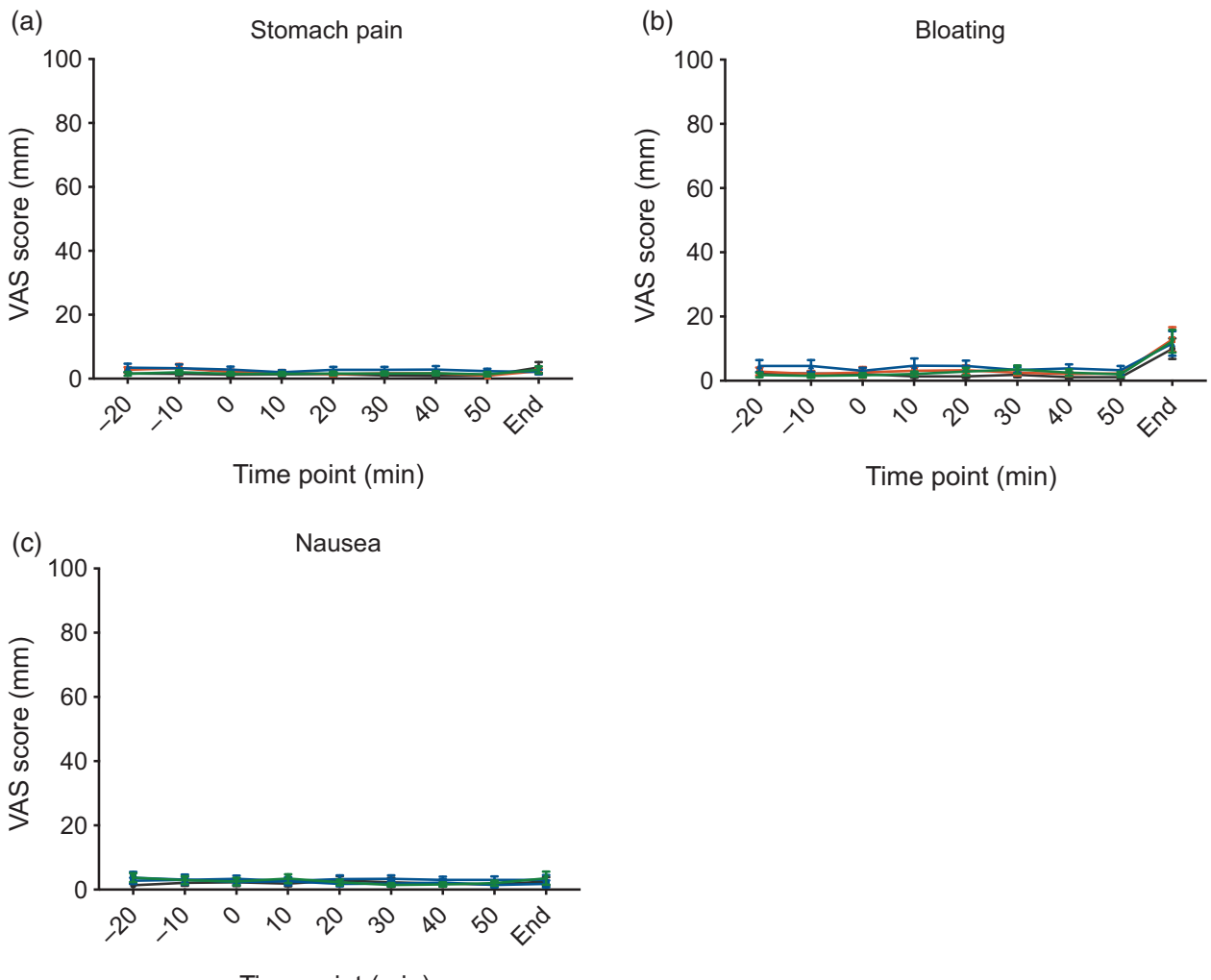

Fig. 5. Visual analogue scale (VAS) scores for stomach pain (a), bloating (b) and nausea (c) (mean + SEM) before and after oral placebo and intragastric placebo (OPGP), oral quinine and intragastric placebo (OQGP), oral placebo and intragastric quinine (OPGQ) and quinine both oral and intragastric (OQGQ). Based on an autoregressive (1) linear mixed model, no differences were observed between conditions. $\rightarrow$, OPGP, $\rightarrow$, OQGP, $\longrightarrow$, OPGQ, $\rightarrow$, OQGQ.
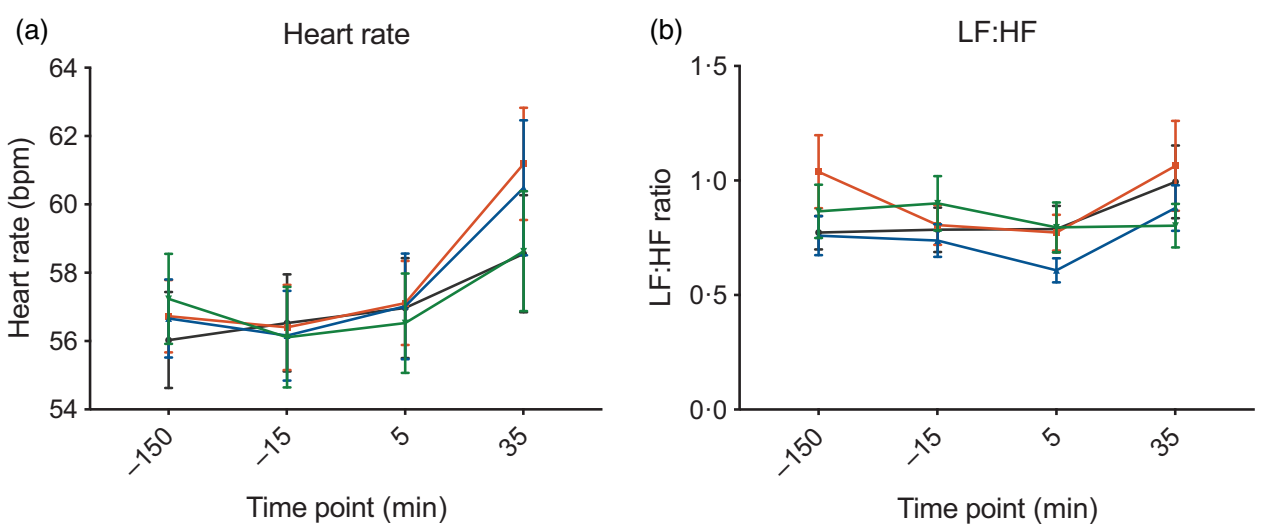

Fig. 6. Heart rate variability (HRV) depicted by heart rate and low-frequency (LF):high-frequency (HF) ratio before and after oral placebo and intragastric placebo (OPGP), oral quinine and intragastric placebo (OQGP), oral placebo and intragastric quinine (OPGQ) and quinine both oral and intragastric (OQGQ). Based on an autoregressive (1) linear mixed model, no differences were observed between conditions. $\rightarrow, \mathrm{OPGP}, \multimap, \mathrm{OQGP}, \longrightarrow, \mathrm{OPGQ}, \multimap, \mathrm{OQGQ}$.

unexpected finding could be explained by the higher starting point for this intervention, influencing a possible overall intervention effect.

Contrary to desire to eat and hunger sensations, satiation and fullness sensations were not affected. Since there was no intervention effect on actual food intake, this raises the question whether actual food intake might be more dependent on satiation and fullness rather than desire to eat and hunger. In addition, energetic intake might be influenced by a large array of different sensations and factors that are not necessarily captured by a single VAS score. Various studies that did find a significant decrease in food intake after GI delivery of tastants showed mixed results on VAS scores for appetite sensations. Iven et al. ${ }^{(34)}$ showed a decrease in hunger and an increase in satiety and fullness. Van Avesaat et al. ${ }^{(16)}$ found a decrease in desire to eat and hunger and an increase in satiety, but no effects on fullness. Andreozzi et al. found no differences in satiation or desire to eat scores ${ }^{(33)}$. It must be noted that in the present study, no intervention $\times$ time interaction was found and therefore conclusions on timing of the effect of oral sham feeding with- and/or 
intragastric delivery of quinine on appetite sensations cannot be drawn. Moreover, interpretation of VAS scores for appetite sensations should always be done with care, since this usually is a secondary outcome parameter in studies on food intake and most studies might not have been adequately powered for such outcomes - as is the case for our study.

A strength of the design of the present study is the large sample size when compared with other research in the field. Moreover, possible inter-subject confounding factors (i.e. habitual meal size, daily energy requirements) were countered by the crossover design. However, this study also has several limitations. A limitation of this study is generalisability to daily life. In this study, subjects consumed their test meal without external stimuli (i.e. telephone use, watching television) and were asked to stop eating when comfortably full. In daily life, people might not be focused on their appetite sensations and be distracted by said external stimuli, which are known to have an increasing effect on eating behaviour ${ }^{(39,40)}$. However, before implementing interventions in daily life, their effects should first be investigated under controlled conditions, as was done in the present study. Another important limitation of this study is that systemic GI peptide levels were not assessed. Measurement of GI peptides could have provided a more complete overview of the effect of oral sham feeding with- and/or intragastric delivery of quinine on eating behaviour. However, a previous study from our group showed a decrease in food intake and increase in satiation without marked changes in systemic GI peptide levels ${ }^{(16)}$. Although in vitro stimulation of intestinal cell lines with tastants results in markedly increased levels of GI peptides, these results have not always been reproduced in vivo, as stated above. It should be noted that all studies measured plasma levels of GI peptides, in search for systemic effects. It remains to be determined what the effect of GI delivery of tastants is on local splanchnic excretion of GI peptides or on possible paracrine effects. Measurement of local levels of GI peptides requires complex invasive techniques. We focused on other, non-invasive, markers that can indicate a shift in hunger/satiation, such as the balance in ANS, which can occur before systemic GI peptide changes can be detected ${ }^{(19,20)}$ and used HRV measurements ${ }^{(23)}$.

In the present study, no differences in HRV measurements were observed between various interventions, which is in line with the fact that there was no shift in hunger/satiation, as indicated by the VAS scores for fullness and satiation. Moreover, food intake was not influenced by the various interventions. A recent meta-analysis showed that $\mathrm{HRV}$ is sensitive to changes in the ANS as a response to stress ${ }^{(41)}$. Although two studies showed meal intake to influence $\mathrm{HRV}^{(42,43)}$, it is uncertain whether HRV measurements are sensitive enough to detect satiation responses. Therefore, future studies should ascertain whether the application of HRV measurements in detecting satiation responses has an added value.

Furthermore, it has to be noted that the ideal interval between tastant delivery and the timing of the ad libitum test meal is unknown. In the present study, we employed a timing identical to that of studies from other groups investigating the effects of bitter compounds on food intake ${ }^{(29,30,33)}$. However, these studies investigated only intragastric or intraduodenal effects of bitter tastants. It is possible that effects of oral sham feeding with quinine could have been found if the test meal was offered sooner after the intervention, since tasting a substance orally might have more acute effects. Moreover, our results suggest a fast response on desire to eat and hunger scores after intervention which could have driven the overall effect that was found. It is possible that earlier presentation of the test meal would have resulted in a reduction in energy intake after administration of quinine. On the other hand, the possibility of an increase in energy intake, due to the need to quell their symptoms (i.e. get rid of the bad taste of quinine), cannot be excluded. Future research protocols should consider timing as a factor to be investigated.

Another important aspect to mention is the dosage of quinine. According to the code of federal regulations stated by the United States Food and Drug Administration, soft drinks such as tonic water are allowed to contain eighty-three parts per million quinine ${ }^{(44)}$. In this protocol, we chose to administer $75 \mathrm{mg}$ of quinine-hydrochloride which is lower than this amount and aligns with previous studies conducted by our $\operatorname{group}^{(16,17)}$. Due to this relatively low dosage of quinine, should we have found significant effects on eating behaviour, this dosage of quinine could have been easily implemented for daily intake. Other studies employ various dosages of quinine administration ranging from 18 to $600 \mathrm{mg}^{(29,30,32-34,36)}$. The higher dosages may give rise to medical problems when implemented for daily intake. A systematic review on the usage of quinine as treatment for muscle cramps showed an increase in minor adverse events such as GI complaints, headache and tinnitus after daily intake of 200-500 $\mathrm{mg}$ for $3 \mathrm{~d}$ up to several weeks ${ }^{(45)}$. This aspect should also be considered when investigating the effects on eating behaviour of higher dosages of quinine.

Up to now, most studies investigating the effects of tastants on food intake focused on acute effects in single test meals. Repetitive or chronic administration of tastants has not been investigated, warranting studies to be conducted on long-term effects of tastants on energy intake.

In conclusion, we have shown that oral sham feeding with- or intragastric delivery of the bitter tastant quinine decreased desire to eat and hunger feelings, without affecting food intake, feelings of satiation and fullness, and HRV when compared with placebo.

\section{Acknowledgements}

We thank all the volunteers for participating in this study.

This research received no specific grant from any funding agency, commercial or not-for-profit sectors. Affiliation 1 is supported by the Province of Limburg, The Netherlands.

The authors' responsibilities were as follows: formulating research questions: T. K., D. K., A. B., A. A. M. M. and F. J. T.; designing the study: T. K., D. K., A. M. E. A., E. W., A. B., A. A. M. M. and F. J. T.; investigation: T. K. and A. M. E. A.; data analysis: T. K.; writing-original draft preparation: T. K.; writing review and editing: T. K., A. M. E. A., E. W., D. K., A. A. M. M. and F. J. T. 
The researchers were previously involved in an investigatorinitiated study on a similar topic ${ }^{(17)}$ for which Will Pharma SA received governmental funding (Subsidie MKB Innovatiestimulering Topsectoren, MIT) and covered all relevant costs related to the execution of that study. T. K. received a salary from Will Pharma SA as part of the 'Subsidie MKB Innovatiestimulering Topsectoren' (MIT) for the period related to the execution of that study ${ }^{(17)}$. D. K. and A. A. M. M. have received an unrestricted grant from Will Pharma SA for the execution of a study unrelated to the present study (peppermint oil in IBS). D. K. and A. A. M. M. have received grants from Zon MW, The Netherlands Organisation for Health Research and Development, to evaluate efficacy of peppermint oil in IBS, hypnotherapy in IBS, and the efficacy of nortriptyline in functional dyspepsia. D. K. and A. A. M. M. have received research funding from Allergan and Grünenthal on IBS topics. D. K. has received research funding from the Dutch Foundation for Gastroenterology (Maag-LeverDarmstichting) for IBS and United Europe Gastroenterology (UEG) for faecal incontinence. A. A. M. M. has given scientific advice to Bayer (topic: IBS), to Kyowa Kirin (topic: constipation), and to Takeda (topic: gastroparesis). A. A. M. M. received funding from Pentax Europe GmbH. A. A. M. M. has received funding from the Dutch Cancer Society related to endoscopy and to colorectal polyps. A. M. E. A., E. W., A. B., and F. J. T. reported no conflicts of interest.

\section{References}

1. WHO (2018) Obesity and overweight. https://www.who. int/en/news-room/fact-sheets/detail/obesity-and-overweight (accessed December 2019).

2. Verdich C, Flint A, Gutzwiller JP, et al. (2001) A meta-analysis of the effect of glucagon-like peptide-1 (7-36) amide on ad libitum energy intake in humans. J Clin Endocrinol Metab 86, 4382-4389.

3. Lieverse RJ, Jansen JB, Masclee AA, et al. (1995) Satiety effects of a physiological dose of cholecystokinin in humans. Gut $\mathbf{3 6}$, 176-179.

4. Lieverse RJ, Jansen JB, van de Zwan A, et al. (1993) Effects of a physiological dose of cholecystokinin on food intake and postprandial satiation in man. Regul Pept 43, 83-89.

5. van Avesaat M, Troost FJ, Ripken D, et al. (2015) Ileal brake activation: macronutrient-specific effects on eating behavior? Int $J$ Obes 39, 235-243.

6. Maljaars PW, Peters HP, Mela DJ, et al. (2008) Ileal brake: a sensible food target for appetite control. A review. Physiol Behav 95, 271-281.

7. Shin HS, Ingram JR, McGill AT, et al. (2013) Lipids, CHOs, proteins: can all macronutrients put a 'brake' on eating? Physiol Behav 120, 114-123.

8. Alleleyn AM, van Avesaat M, Troost FJ, et al. (2016) Gastrointestinal nutrient infusion site and eating behavior: evidence for a proximal to distal gradient within the small intestine? Nutrients $\mathbf{8}, 117$.

9. van der Wielen N, van Avesaat M, de Wit NJ, et al. (2014) Crossspecies comparison of genes related to nutrient sensing mechanisms expressed along the intestine. PLOS ONE 9, e107531.

10. Young RL, Sutherland K, Pezos N, et al. (2009) Expression of taste molecules in the upper gastrointestinal tract in humans with and without type 2 diabetes. Gut 58, 337-346.
11. Bezencon C, le Coutre J \& Damak S (2007) Taste-signaling proteins are coexpressed in solitary intestinal epithelial cells. Chem Senses 32, 41-49.

12. Sternini C, Anselmi L \& Rozengurt E (2008) Enteroendocrine cells: a site of 'taste' in gastrointestinal chemosensing. Curr Opin Endocrinol 15, 73-78.

13. Jang HJ, Kokrashvili Z, Theodorakis MJ, et al. (2007) Gutexpressed gustducin and taste receptors regulate secretion of glucagon-like peptide-1. Proc Natl Acad Sci U S A 104, 15069-15074.

14. Rozengurt N, Wu SV, Chen MC, et al. (2006) Colocalization of the alpha-subunit of gustducin with PYY and GLP-1 in L cells of human colon. Am J Physiol Gastrointest Liver Physiol 291, G792-G802.

15. Geraedts MC, Troost FJ \& Saris WH (2011) Different tastants and low-caloric sweeteners induce differential effects on the release of satiety hormones. Food Chem 129, 731-738.

16. van Avesaat M, Troost FJ, Ripken D, et al. (2015) Intraduodenal infusion of a combination of tastants decreases food intake in humans. Am J Clin Nutr 102, 729-735.

17. Klaassen T, Alleleyn AME, van Avesaat M, et al. (2019) Intraintestinal delivery of tastants using a naso-duodenal-ileal catheter does not influence food intake or satiety. Nutrients 11, 472 .

18. Breslin PA \& Spector AC (2008) Mammalian taste perception. Curr Biol 18, R148-R155.

19. Nederkoorn C, Smulders FT \& Jansen A (2000) Cephalic phase responses, craving and food intake in normal subjects. Appetite 35, 45-55.

20. Mattes RD (1997) Physiologic responses to sensory stimulation by food: nutritional implications. J Am Diet Assoc 97, 406-413.

21. Harthoorn LF \& Dransfield E (2008) Periprandial changes of the sympathetic-parasympathetic balance related to perceived satiety in humans. Eur J Appl Physiol 102, 601-608.

22. Pavli F, Tassou C, Nychas GE, et al. (2018) Probiotic incorporation in edible films and coatings: bioactive solution for functional foods. Int J Mol Sci 19, 150.

23. Anonymous (1996) Heart rate variability. Standards of measurement, physiological interpretation, and clinical use. Task Force of the European Society of Cardiology and the North American Society of Pacing and Electrophysiology. Eur Heart $J$ 17, 354-381.

24. Burdock GA (2007) Safety assessment of hydroxypropyl methylcellulose as a food ingredient. Food Chem Toxicol 45 , 2341-2351.

25. Steiner JE, Glaser D, Hawilo ME, et al. (2001) Comparative expression of hedonic impact: affective reactions to taste by human infants and other primates. Neurosci Biobehav Rev 25, 53-74.

26. Just T, Pau HW, Engel U, et al. (2008) Cephalic phase insulin release in healthy humans after taste stimulation? Appetite $\mathbf{5 1}$, 622-627.

27. Teff KL, Devine J, Engelman K (1995) Sweet taste: effect on cephalic phase insulin release in men. Physiol Behav 57, 1089-1095.

28. Parker BA, Sturm K, MacIntosh C, et al. (2004) Relation between food intake and visual analogue scale ratings of appetite and other sensations in healthy older and young subjects. Eur J Clin Nutr 58, 212-218.

29. Deloose $\mathrm{E}$, Janssen $\mathrm{P}$, Corsetti M, et al. (2017) Intragastric infusion of denatonium benzoate attenuates interdigestive gastric motility and hunger scores in healthy female volunteers. $\mathrm{Am}$ J Clin Nutr 105, 580-588.

30. Bitarafan V, Fitzgerald PCE, Little TJ, et al. (2019) Effects of intraduodenal infusion of the bitter yastant, quinine, on antropyloroduodenal motility, plasma cholecystokinin, and energy intake in healthy men. J Neurogastroenterol Motil 25, 413-422. 
31. Peters HPF, Koppenol W, Schuring EAH, et al. (2016) The effect of two weeks ingestion of a bitter tastant mixture on energy intake in overweight females. Appetite 107, 268-273.

32. Bitarafan V, Fitzgerald PCE, Little TJ, et al. (2020) Intragastric administration of the bitter tastant quinine lowers the glycemic response to a nutrient drink without slowing gastric emptying in healthy men. Am J Physiol Regul Integr Comp Physiol 318, R263-R273.

33. Andreozzi P, Sarnelli G, Pesce M, et al. (2015) The bitter taste receptor agonist quinine reduces calorie intake and increases the postprandial release of cholecystokinin in healthy subjects. J Neurogastroenterol Motil 21, 511-519.

34. Iven J, Biesiekierski JR, Zhao D, et al. (2019) Intragastric quinine administration decreases hedonic eating in healthy women through peptide-mediated gut-brain signaling mechanisms. Nutr Neurosci 22, 850-862.

35. Mennella I, Fogliano V, Ferracane R, et al. (2016) Microencapsulated bitter compounds (from Gentiana lutea) reduce daily energy intakes in humans. Br J Nutr 116, 1841-1850.

36. Deloose E, Corsetti M, Van Oudenhove L, et al. (2018) Intragastric infusion of the bitter tastant quinine suppresses hormone release and antral motility during the fasting state in healthy female volunteers. Neurogastroenterol Motil 30, e13171.

37. Little TJ, Gupta N, Case RM, et al. (2009) Sweetness and bitterness taste of meals per se does not mediate gastric emptying in humans. Am J Physiol Regul Integr Comp Physiol 297, R632-R639.

38. van den Akker K, Havermans RC \& Jansen A (2017) Appetitive conditioning to specific times of day. Appetite 116, 232-238.

39. Boulos R, Vikre EK, Oppenheimer S, et al. (2012) ObesiTV: how television is influencing the obesity epidemic. Physiol Behav 107, 146-153.

40. Ogden J, Coop N, Cousins C, et al. (2013) Distraction, the desire to eat and food intake. Towards an expanded model of mindless eating. Appetite 62, 119-126.

41. Kim HG, Cheon EJ, Bai DS, et al. (2018) Stress and heart rate variability: a meta-analysis and review of the literature. Psychiatry Investig 15, 235-245.

42. Harthoorn LF \& DransWeld E (2008) Periprandial changes of the sympathetic-parasympathetic balance related to perceived satiety in humans. Eur J Appl Physiol 102, 601-608.

43. Sauder KA, Johnston ER, Skulas-Ray AC, et al. (2012) Effect of meal content on heart rate variability and cardiovascular reactivity to mental stress. Psychophysiology 49, 470-477.

44. US Food and Drug Administration (2019) CFR - Code of Federal Regulations Title 21. https://www.accessdata.fda.gov/scripts/ cdrh/cfdocs/cfCFR/CFRSearch.cfm?fr $=172.575$ (accessed December 2019).

45. El-Tawil S, Al Musa T, Valli H, et al. (2015) Quinine for muscle cramps. Cochrane Database Syst Rev, issue 4, CD005044. 